\title{
Wireless Sensor Data Acquisition and Control Monitoring Model for Internet of Things Applications
}

\author{
SulaimaLebbe Abdul Haleem, ${ }^{1}$ Pravin R. Kshirsagar (iD, ${ }^{2}$ Hariprasath Manoharan, \\ BoppuruRudra Prathap, ${ }^{4}$ Hemalatha $S,{ }^{5}$ Kukatlapalli Pradeep Kumar, ${ }^{5}$ Vineet Tirth, ${ }^{6}$ \\ Saiful Islam, ${ }^{7}$ Raghuveer Katragadda, ${ }^{8}$ and Temesgen Abeto Amibo ${ }^{9}{ }^{9}$ \\ ${ }^{1}$ Department of Information \& Communication Technology, South Eastern University of Sri Lanka (SEUSL), Oluvil, Sri Lanka \\ ${ }^{2}$ Department of Artificial Intelligence, G. H. Raisoni College of Engineering, Nagpur 440016, India \\ ${ }^{3}$ Department of Electronics and Communication Engineering, Panimalar Institute of Technology, Poonamallee, Chennai, \\ Tamil Nadu, India \\ ${ }^{4}$ Department of Computer Science and Engineering, CHRIST University, Bangalore, India \\ ${ }^{5}$ Department of Computer Science and Engineering, Panimalar Institute of Technology, Chennai, India \\ ${ }^{6}$ Mechanical Engineering Department, College of Engineering, King Khalid University, Abha 61411, Asir, Saudi Arabia \\ ${ }^{7}$ Civil Engineering Department, College of Engineering, King Khalid University, Abha 61411, Asir, Saudi Arabia \\ ${ }^{8}$ Department of Business Management, V. R. Siddhartha Engineering College, Vijayawada, Andhra Pradesh, India \\ ${ }^{9}$ School of Chemical Engineering, Jimma Institute of Technology, Jimma University, Jimma, Oromia, Ethiopia
}

Correspondence should be addressed to Pravin R. Kshirsagar; pravinrkshirsagarphd@gmail.com and Temesgen Abeto Amibo; temesgen.abeto@ju.edu.et

Received 20 December 2021; Revised 7 January 2022; Accepted 13 January 2022; Published 17 February 2022

Academic Editor: M Pallikonda Rajasekaran

Copyright (c) 2022 SulaimaLebbe Abdul Haleem et al. This is an open access article distributed under the Creative Commons Attribution License, which permits unrestricted use, distribution, and reproduction in any medium, provided the original work is properly cited.

\begin{abstract}
This article focuses on providing solutions for one important application termed as agriculture. In India, one major occupation for people living in urban and rural areas is agriculture where an economic rate depends only on the crops they yield. In such cases, if an intelligent monitoring device is not integrated then it becomes difficult for the farmers to grow their crops and to accomplish marginal income from what they have invested. Also existing methods have been analyzed in the same field where some devices have been installed and checked for increasing the productivity of horticulture crops. But existing methods fail to install an intelligent monitoring device that can provide periodic results within short span of time. Therefore, a sensor based technology with Internet of Things (IoT) has been implemented in the projected work for monitoring major parameters that support the growth and income of farmers. Also, an optimization algorithm for identifying the loss in different crops has been incorporated for maximizing the system boundary and to transmit data to farmers located in different areas. To prove the cogency of proposed method some existing methods have been compared and the results prove the projected technique produces improved results for about $58 \%$.
\end{abstract}

\section{Fundamental Implementation of Sensors and IoT}

In this section fundamental approach of sensors and Internet of Things (IoT) has been deliberated, where a human operation is completely replaced by intelligent monitoring devices. When such intelligent devices are incorporated it is necessary to implement a cloud based solution and this is possible using IoT where all data is distributed in wireless mode to each individual within a short period of time. Sensors, an intelligent wireless module, have been introduced as a replacement for existing methods that use laboratory equipment without any wireless module. In this present circumstance sensors 
and IoT have been used in various applications such as medical care, automobiles, transportation, etc. for providing immediate monitored results to corresponding central station where all parametric results will be coordinated in a similar fashion that is understandable by different users. This kind of devices can also be extended for installing in ad hoc mode where even with varying nodes the users can be able to develop parametric results for all different applications.

In recent period the intelligent devices are also installed with USB based interface with single board capability where high power will be equal to 5 volts. This type of intelligent device saves much high power during the output operating profile and by having such wireless module in indicator all high end boards such as Raspberry Pi, Arduino, and embedded modules can be connected in an easy mode. Since the proposed method focuses on sensor with IoT one important constraint in day-to-day life is security, where this type of devices will offer high security at low cost. In addition, this wireless device can be installed in wearable mode with different online support services.

1.1. Related Works. Many research papers have been analyzed for understanding the integration of sensors in agricultural applications where most of the authors have provided solutions for solving many major issues in their nearby areas. Even [1] has focused on monitoring each stage of crop using an unnamed aerial management system where all crops can be optimized at earlier stages before reaping. This process can be able to grow the crops in a virtuous manner thus providing high income to farmers. But the major drawback is difficulty in monitoring the optimization process of crops without using an intelligent sensing device [1]. Thus the authors have analyzed only recent trends that support the growth on integrating Internet of Things (IoT) in agricultural applications. Also, hydroponic monitoring systems have been developed [2] for controlling all necessary parameters like level of water, humidity, and temperature. These parameters are much essential for all agricultural applications and it can be monitored only through integration of sensing devices which is considered as main disadvantage [2]. A light dependent resistor is used for monitoring all parameters which is having the capability of increasing the light visibility for measuring alkalinity in water. These types of sensors can be able to work in all environmental conditions and they can be able to settle fast, thus providing an artificial lake which is completely controlled by development of mobile applications. Additionally, a control pump is also integrated with ESP 32 microcontroller for taking water directly from artificial lakes. These types of microcontrollers are directly connected to water sensors for monitoring the level of water in the lake. If the level of water is taken much higher than the prescribed amount from the lake then an alarm will go off in that particular field.

For reconciling all the problems that are present in land for vertical farming a review has been conducted by taking reference of various fields [3]. The authors stated that even though much advanced technologies exist in the present trend the main problem is that there is no such system to minimize the utilization of land resources [3]. Therefore, a new method of vertical farming has been introduced in the system where the yield of crops can be increased at much higher extent and net amount can be returned to farmers which is beneficial for those who are having small acres of land. This yield can be made higher by growing many substrates which supports intelligent growth in a particular field. Even the same methodology can be applied to all residential apartments [4] where people can grow healthy vegetables and they can use them for their daily purpose. The risk involved in this type of process is much lesser and at the same time cost of implementation in residential areas is much lesser. Moreover the time of yield is much similar to fields which incorporate urban agriculture. However, the shortcomings are that controlled environmental conditions should be established in the indoor rural environments [4] whenever an IoT process in implemented [5]. Most of the farmers who are having marginal acres of land will not have any mobile phones for monitoring their fields. In this case it is better to implement a web browser which even supports small mobile devices with sufficient Internet facility. These web browsers can be used for monitoring soil moisture on the field and maximum amount of water content can also be observed by using distinct Internet Protocol (IP) address. But some shortage occurs in IoT implementation where local farmers should receive communications in their local language [5].

In addition to different applications that are deliberated overhead the practice of producing food and increasing its sustainability in all controlled environments should be established using technology centric methods [6]. This type of protection in controlled environment will increase the growing structure of crop and if a controlled environment is established then soilless farming can be guaranteed. Nevertheless in real time situation it is a challenging task to establish a controlled environment [6] and it is not possible to enhance the systems using new techniques. A new harvesting technique which involves the process of natural resources using windmill has been introduced [7] for better yielding and for managing different water techniques which is entirely different when compared with [1-6]. The technique introduced for integrating natural resources consists of hydroponics connections which are directly connected to all pipelines but the delinquent detected in [7] is that farmers should connect their pipeline in central system which increases the cost of implementation. Even the same method is possible in vertical cities where production of food and its consumption rate can be stored at the same domicile [8]. Thus economic productivity can be increased to much higher extent, thus decreasing all major obstacles that are present in the growth of vertical farming. In this case if economic productivity is increased due to vertical farming then snag in other stages of farming should be reduced; i.e., old methods of farming can be left out [8]. The authors in [9] have examined that in the near future it is possible to implement innovative farming industries that give base high amount to all farmers located in rural and urban areas. This 
process can be able to reduce scarceness in food, thus adding high safety in production of food. On the other hand percentage of safety is much lesser which lies in the limit between 5 and 10 percentages [9].

If both rural and urban areas are integrated under smart city process then a weather monitoring and control system should be established using sensor networks [10]. A Raspberry Pi module will be integrated in vertical cities for both monitoring and control persistence in all agricultural farms. If a weather monitoring process is present then all necessary environmental parameters should be monitored using a slave controller $[11,12]$ where a Hyper Text Transfer Protocol (HTTP) will be used for sending and receiving the data. Further the usage of natural resources has been extended using a solar panel which is accompanied with water sensors [13-17]. But ancient methods of agriculture, i.e., before the invention of sensors concentration level in crops, are identified using $\mathrm{pH}$ controller [18]. In addition some laboratory equipment has been used for analyzing the results which is very difficult to carry when measurements are needed within short span of time. The foremost entity in existing methods without intelligent device is that diseases in crops cannot be identified in an easy way as the farmers are not having any provision excluding handmade solutions. Apart from the underlying concept integration of different sensors for monitoring the agricultural field has been deliberated [19] with an RFID tag which is used for identifying relevant fields. This type of Radio Frequency Identification (RFID) is connected to antenna systems which is in turn used by the transponder for transmitting and receiving monitored parameters. In the next segment a gateway which is used as a source of connecting different nodes is released for connecting a local network. Then followed by the gateway process three different sensors are integrated for measuring the agricultural lands. This type of integration forms the rudimentary segment for the proposed method for achieving high accurate predicted results. One major drawback in abovementioned method [10-18] is that it focuses on implementing a hardware module such as Raspberry pi and different microcontrollers for monitoring different parameters but a real time based solution in vertical farming can be obtained only after monitoring parameters like height, load, and thickness of crops which is discussed in the subsequent sections. Table 1 describes the assessment of existing and proposed techniques.

1.2. Research Gap and Motivation. Even though many authors have formulated different information based solutions to all problems in farming for different crops still certain gap exists in framing solutions to different parametric objectives such as height, load, and thickness of crops. Also, there are no proper formulations followed for implementing such real time applications using sensors. Therefore, the authors have formulated a unique model for monitoring all necessary parameters that are essential for growth of crops and in turn provide sufficient day-to-day information to all farmers using an online based monitoring system. Even it is possible to send information to all farmers in their local languages which can be canned as one additional advantage of the proposed method.

In addition the proposed work has been motivated after analyzing the difficulties that are faced by farmers in their day-to-day existence where the amount of income which they are earning is being reduced in a cyclic manner. Therefore certain constraints are designed before application of sensors which will provide substantial benefit to all farmers and at the same time a basic pay will be accompanied when farming is introduced with intelligent device for monitoring persistence.

1.3. Objectives. The major objectives of proposed method by implementing intelligent monitoring device are divided in three items as follows:

(i) Design and implementation of real time information based data centric method which can monitor the pinnacle, load, and thickness of crops in farming areas.

(ii) Incorporating a smart sensor based water treatment methodology for measuring the level of water in crops.

(iii) Implementation of soil based sensor devices for monitoring the level of fertilizers and to implement ultrasonic sensors for monitoring different diseases that affect the productivity of crops.

\section{Problem Formulation}

This section describes the mathematical procedure for implementing sensors in required fields where the basic parameters should be predefined inside multiple sensors for achieving equivalent system implementation. Therefore, one major requirement of sensor in agricultural field is to spread proper amount of agrochemicals into the meadow where dosed up amount should be at standard rate because when amount of dosing is higher than estimated amount then crops can be able to grow at much faster rate and natural method of farming cannot be realized in agricultural farms. Therefore, it is necessary to monitor the difference between existing and dispensed dosage amount by using two different settings as given in the following equation:

$$
M_{i}=\sum_{i=1}^{n} V_{i} \times \omega,
$$

where

$V_{i}$ denotes the amount of volume required for agricultural field;

$\omega$ represents the concentration of chemicals that are installed in field.

Equation (1) denotes the amount of material that is delivered to agricultural crops which is denoted as $M_{i}$. The materials can be delivered in the field by following certain amount of thickness and values of concentration will be 
TABle 1: Assessment of existing and proposed techniques.

\begin{tabular}{lccc}
\hline Reference & Technique & Type followed & Contributed parameters \\
\hline$[2]$ & Hydroponic system & Microcontroller based & Energy consumption and cost \\
{$[5]$} & Indoor vertical farming & Microcontroller and Ethernet shield & Energy consumption \\
{$[6]$} & Vertical farm module & AT mega controller & Climate instabilities \\
Proposed & Data centric method at low cost & Sustenance path appliance (SPA) & Pinnacle, load, and thickness of crops \\
\hline
\end{tabular}

monitored by wireless sensors using different considerations as given in the following equation:

$$
E_{i}=\sum_{i=1}^{n} B_{i} R_{i}+\bar{H}(i),
$$

where

$B_{i}$ represents the capacity of battery to be installed;

$R_{i}$ denotes the residual energy supplied to nodes;

$\bar{H}(i)$ signifies actual expanse of harvested energy that is taken from previous instant.

Since a variable in equation (2) depends on previous state values it is necessary to separate weights of each node. Thus equation (2) should be denoted using following constraint:

$$
\bar{H}(i)=\varphi \overline{H_{1}+(1-\varphi) \overline{H_{2}}},
$$

where

$\varphi$ denotes the weight of synchronized process which varies between 0 and 1 ;

$\overline{H_{1}}$ and $\overline{H_{2}}$ represent the harvested energy of first and second zones, respectively.

The process of agricultural farming in large acres of land will have high amount of materials that are supplied for delivering fertilizers by considering accurate timing parameters for increasing the quality of monitoring. Thus bulk materials that are supplied for fertilizers through buzz monitoring can be given as

$$
B_{F}(i)=\sum_{i=1}^{n} E_{i} \times t_{i}
$$

where

$B_{F}(i)$ denotes delivering bulk amount of fertilizers;

$t_{i}$ signifies the amount of time taken for delivering majority fertilizers.

Equation (4) indicates that high amount of fairness should be present with maximized network coverage. Therefore if maximum values are achieved it is much easier to get advanced connectivity thus monitoring high amount of fertilizers in large acres of land. The same constraint can be formulated as

$$
\left|f_{1}(i)-f_{2}(i)\right|<d_{i}
$$

where

$f_{1}(i)$ and $f_{2}(i)$ denote the frequency components of first and second target nodes, respectively; $d_{i}$ represents the distance of separation between two nodes.

Equation (5) indicates that frequency components should always be lesser than distance of separation, i.e., difference between two different nodal configurations during monitoring cycle. In some cases if distance of separation is higher than expected computation amount then, entire frequency components have to be restored without any loss of data. The network connectivity for monitoring persistence will be much higher if such strategy as denoted in equation (5) is followed.

If constraint in equation (5) is gratified then ratio of nitrogen, phosphorous, and potassium (NPK) should be designed for sheeting down the chemicals equally. For this equal scattering process the density of fertilizers which is reserved in greater part from equation (4) should be considered. Therefore, the amount of NPK density should be monitored using equation (6) as follows:

$$
\mathrm{NPK}_{i}=\sum_{i=1}^{n}\left(V_{i} \omega\right)\left(t_{i} S_{i}\right)
$$

where $S_{i}$ represents the solidity content of NPK, respectively.

In equation (6) the content of NPK comes with equal concentration from different fertilizers; therefore the solidity content which is calculated should be equal to proportion of nitrogen inside the fertilizer. If proportion is not equal then more variation in growth of a particular crop will be sensed by sensors. This creates error in sensing process which can be formulated in mathematical form as

$$
M(i)=\sum_{i=1}^{n} \sqrt{S_{1}^{2}+S_{2}^{2}+S_{3}^{2}+\cdots+S_{n}^{2}},
$$

where $S_{1 \ldots} S_{n}$ denotes the error content in proportion process that is monitored by sensors.

\section{Optimization Algorithm}

Formulation from equations (1)-(7) is considered for both small and large scale farming where the main part will be controlled by wireless sensors for monitoring data. Therefore a machine learning algorithm will be integrated for optimizing development of different crops. Also, different machine learning algorithms have been compared with each other and they are having distinct benefits and difficulties. Therefore, after examining certain investigations in field it is found that Sustenance Path Appliance (SPA) which is an extended version of support vector machine using binary numbers $\{0,1\}$ can be selected because SPA is having the ability to perform better operation even when unstructured 
data is present. Also even if semistructured data using images are fed as an input then a grain hoax methodology will be followed for implementing high dimensional data. In addition one major hazard that persists in all types of machine learning algorithm which is defined as overfitting of data is overwhelmed when SPA is integrated. Also the margin level of SPA is dissimilar to other machine learning algorithms where the boundary will be defined between $[-1$, 1]. The main purpose of integration is to maximize the system boundary which can be expressed in mathematical form as

$$
b_{i}= \begin{cases}0, & \text { if } x_{i} p(i) \geq 1, \\ 1-x_{i} p(i), & \text { else }\end{cases}
$$

where $x_{i} p(i)$ represents the loss function.

Equation (8) denotes that loss function is needed in case that the margins are not maximized because whenever a sensor is placed the margins at four different ends should be enlarged. This type of margin enlargement will prevent the land from different diseases as all boundaries have been monitored and sensed in a regular way. In case of there is no possibility of maximizing the margin level then at least the boundaries should be balanced. But it is difficult to balance all the boundaries every time whenever a sensor is implemented. In such cases one alternate solution will be provided by upgrading the value of gradients. This apprising can be given as

$$
\theta_{i-1}=\theta_{i}-\omega\left(2 \delta_{i}\right)
$$

where $\theta_{i-1}$ and $\theta_{i}$ denote the regularization parameter without any misclassification.

In equation (9) weights of all gradient parameters are considered with a marginal level of 2 . The value of 2 is derived from partial derivative of all weights that are considered in the maximization process. In addition to weight a bias level will be considered for adding it in equation (8) in the form of hyperplane. The addition process can be defined mathematically using the following equation:

$$
V_{i}+b_{i}=0
$$

where

$V_{i}$ represents the set of vectors with incorporation of weights

$b_{i}$ denotes the bias for all corresponding directions

The cogency of proposed method can be proved by observing the efficiency of simulation results in all five different scenarios as discussed in Section 4. When sensor is implemented in real time environmental problems under different applications such as healthcare, transportation, agriculture, and automobiles many parameters have to be evaluated and an efficient algorithm should be integrated for achieving high efficient results. In the proposed method parameters such as water, temperature, and moisture level should be monitored in agricultural environment by integrating three different sensors with level switches. In agricultural applications an optimization algorithm plays an important role for integration purpose where utility process of SPA with IoT is illustrated in Figure 1.

From Figure 1 it can be observed that step-by-step implementation of SPA which is integrated with IoT is provided where at first stage of implementation foregoing parametric values on temperature, moisture, and humidity content are initialized. Once the values are initialized minimum and maximum levels of monitoring parameters can be observed clearly so that the input parameters can be reserved within the observed limit. The description of the sensor values is described in Table 2 .

If any changes are observed with previous and existing method it will be calculated with equation (1) with different sceneries. After certain period of time the concentration level of sensors can be observed using equation (2) which is completely based on capacity of batteries inside the sustenance. If any changes are observed in this segment then different weights are loaded with substance materials which are calculated using equations (3) and (4). Suppose if maximum soil moisture is extended the loop can be clogged but if maximum level is not achieved then level of water should be increased and at final stage disease detection can be identified by integrating the sensors in wireless mode. Since the sensors are installed in wireless mode data will be transferred using cloud infrastructure which is possible with IoT based process by integrating SPA. Since in proposed method a separate optimization algorithm which is indicated as SPA is combined with intelligent sensing device it is not necessary to integrate statistical values with set of training data. As a result even if the data is hidden it can be found accurately at low error rate capability. This is possible in SPA even if training set is much higher because each and every time if data needs to be trained it will be a time consuming process. Therefore to avoid this concern remaining parts are maximized. Further the data in proposed method does not start with noise constraint, thus making the overfitting approach to be a better solution for agro-based solutions.

\section{Results and Discussions}

The proposed work aims to implement a hardware platform using Arduino UNO where the data from Arduino UNO will be read by Raspberry Pi. The programming structure is formed by incorporating Python codes and the final output will be integrated using a serial USB connection where the results are executed using MATLAB. Since more amount of water wastage is observed in agricultural applications in the future a sensor based treatment methodology should be introduced for controlling the amount of water wastage. Therefore, to measure the amount of water level, water sensors are integrated with Arduino where continuous monitoring of $\mathrm{pH}$ (Potential of Hydrogen) level is probable and it will be updated in IoT database. The same setup will be integrated in ThingSpeak and results of the same process will be cross verified; i.e., the same setup can be integrated in both online and offline analysis by considering the following scenarios.

Scenario 1. Extrapolation of pinnacle. 


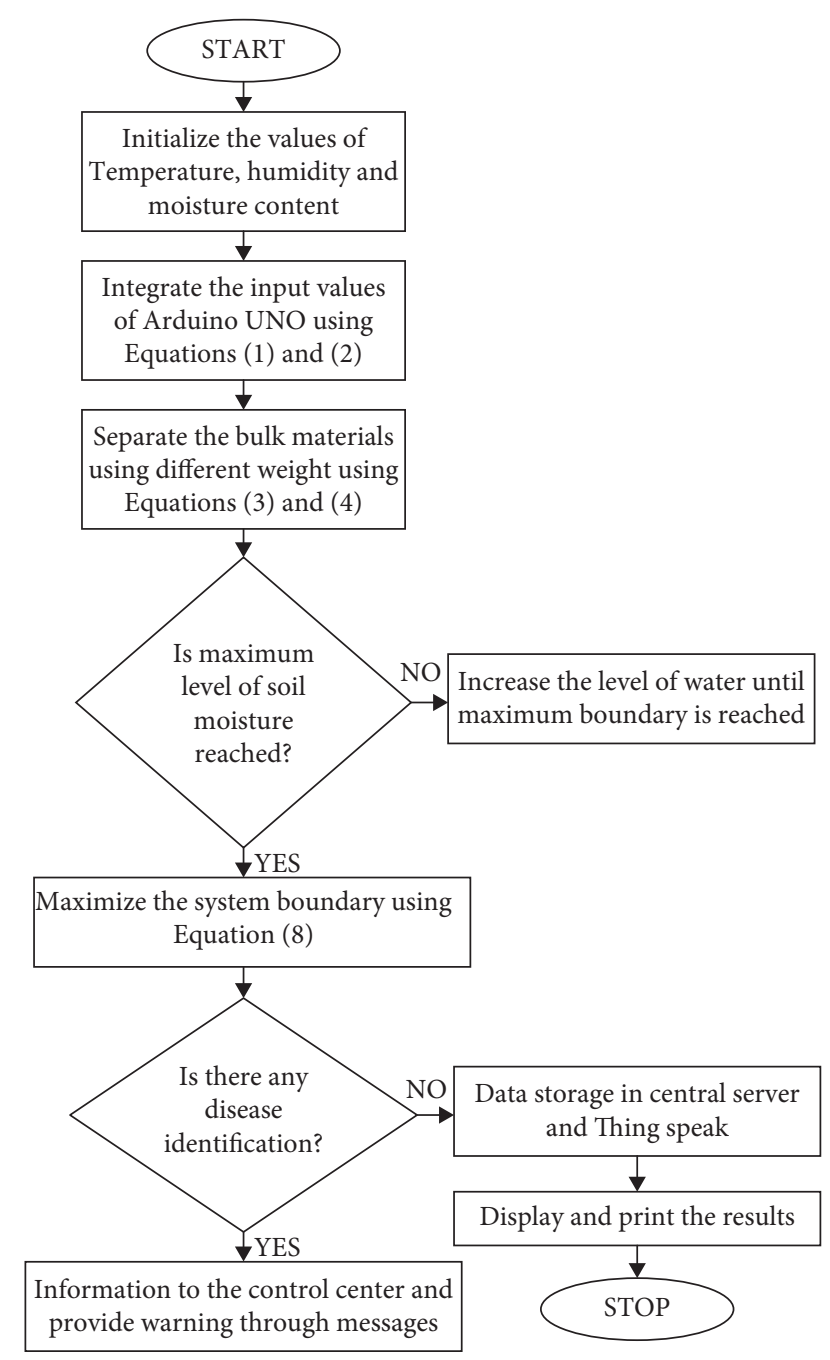

FIGURE 1: Implementation of proposed method using SPA.

Scenario 2. Likelihood of diverse loads.

Scenario 3. Thickness level of crops.

Scenario 4. Energy consumption of sensors.

Scenario 5. Calculation of cost.

In the proposed method three different sensors are integrated where threshold limits (between 0 and 1) are provided in first segment. In second segment predictive values will be monitored and they are stored in IoT cloud using an encrypted key. At central coordination system reference and predicted values are compared and if any changes are observed then they will be reported to farmers using data centric approach using automatic uploading mode. The data stored in central system will be used for standby operation as high storage capacity is provided for data change in a single loop. This data will always be used for significant reference in all agricultural central organizations for detecting the probability of failure. Table 3 summarizes the outputs based on the list of sensors deployed in the environment as follows.
4.1. Scenario 1. In this scenario the foremost parameter for implementing sensors which is labeled as displacement or measurement sensor is integrated and measurements have been made in the proposed area. It is very important to monitor the height of crops because if height of crops falls below a certain level then it can be informed to corresponding farmers and immediate measures can be taken within short span of time. But if the height of crop is measured at late instant of time then it is very difficult to reap the crop during correct period. Thus a sensor is needed to monitor the pinnacle of all the crops and the sensed height of crops is shown in Figure 2. It should always be illustrious that minimum and maximum height of each layer should be between $400 \mathrm{~cm}$ and $800 \mathrm{~cm}$, respectively.

From Figure 2 it can be seen that the number of sensors is taken from 100 to 900 . For each different sensor corresponding heights of crops are monitored and they are compared with proposed method [17]. Figure 2 implies that before implementing sensors height of each layer for existing [17] method is $321 \mathrm{~cm}$ which is much below the prescribed level and it is calculated indiscriminately but when the sensors are implemented for the same area then exact height of crops is found to fall $45 \mathrm{~cm}$ inside the boundary value. Therefore, if sensors are integrated then the exact height can be monitored and the crops can be cultivated at correct periods.

4.2. Scenario 2. In this scenario loads that needed to be allocated for corresponding crops have been estimated. Once the height of each crop is found then loads from each side of the crops should be calculated from equation (4). It is obvious that loads of each crop can be monitored only when intelligent sensing devices are installed because each crop cannot grow at different weights and in turn each farmer can be able to get returns only when weight of each crop is increased to considerable amount. Therefore if sensing devices are installed then fertilizers that are drizzled in crops should be poured in equal amounts. Thus in the proposed method one acre of land is considered where according to variations in height, diverse nodes are distributed and the loads are predicted. All observed values through online software using node red are plotted using MATLAB and are plotted in Figure 3.

It can be observed from Figure 3 that pinnacles that are observed from scenario 1 are considered as reference for calculating load values at each end point in one acre of land. By seeing the height of each crop between minimum and maximum level nodes will be distributed and loads will be predicted by each sensing unit. Figure 3 indicates the comparison of predicted loads in proposed method with existing methods [17] where the installed sensor unit in proposed method outperforms the existing method in such cases when no sensing units are incorporated. The real time implementation values differ much from observed level and it can be apprehended that using less weights the farmers can be able to achieve the same quality of crops with high marginal income but continuous sensing measures are essential. 
TABLE 2: Sensors description.

\begin{tabular}{lc}
\hline Sensor name & Description \\
\hline $\begin{array}{l}\text { Moisture } \\
\text { Humidity } \\
\text { Temperature }\end{array}$ & Refers to the presence of water \\
\hline
\end{tabular}

TABLE 3: Sensors alerts description.

\begin{tabular}{lccc}
\hline Moisture & Temperature & Humidity & Output values \\
\hline Mild & Severe & Moderate & Good \\
Mild & Severe & Below moderate & Good \\
Severe & Low & Low & Alert \\
Severe & Below low & Below low & Alert \\
\hline
\end{tabular}

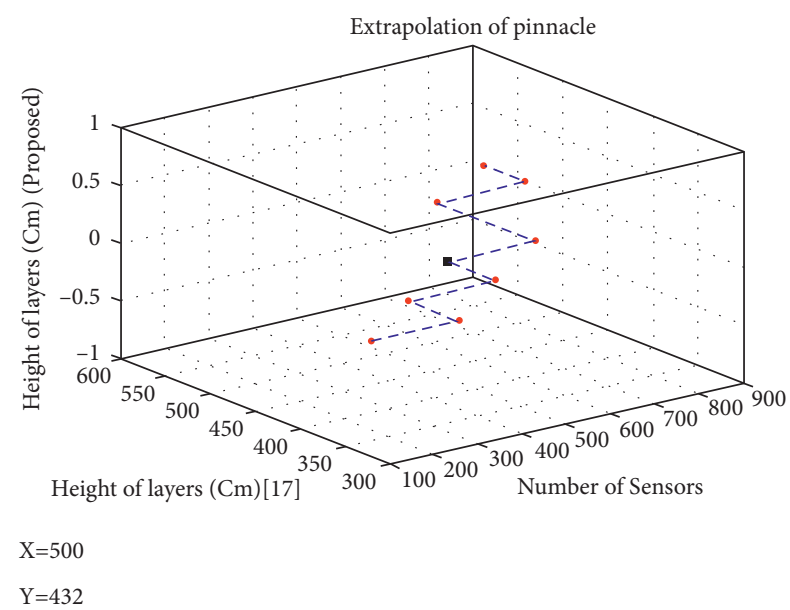

FIgURE 2: Exploration of pinnacle for effected areas.

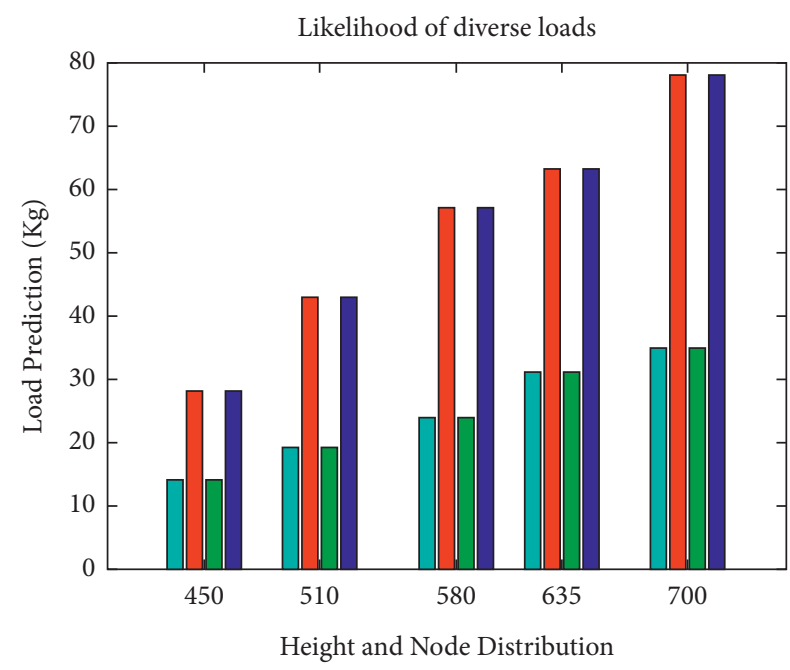

Figure 3: Predicted load for distributed nodes.

4.3. Scenario 3. Once loads are properly allocated for each crop then thickness level of each crop should be calculated using equation (6). All observations made using equation (6) should contain equal percentage of NPK content so that thickness level of crops will be much higher than average exertion level. Thickness in various sheets can be monitored using an ultrasonic sensor where thickness level can be exactly observed. This scenario is much important because during each season the harvesting will be steered depending only on the amount of thickness in each crop; therefore ultrasonic sensors will be placed in the field for monitoring thickness levels of crops. The observed thickness level is plotted in Figure 4 which is measured in microns.

From Figure 4 it can be observed that thickness levels of crops are plotted by using the heights that are observed from Scenario 1. Also, number of acres in this case is considered to be 5 because for small acres of land the amount of thickness will not be constant; therefore for better analysis marginal acres of land are considered. It can be observed from Figure 4 that thickness level of crops is exactly predicted and it is equal to the amount where it can be yielded. For example if height of crops is equal to 580 and $700 \mathrm{~cm}$ then thickness levels are matched to 0.48 and 0.92 microns, respectively. This proves that thickness levels of crops are monitored correctly and high amount of crops can be yielded at the same thickness levels.

4.4. Scenario 4. In this scenario, energy consumption of nodes which can be monitored after exact load prediction is analyzed and the corresponding simulated values are plotted in Figure 5. During simulation part the time necessary for allocating a particular load is also calculated because as time varies the nodal energy consumption will also differ. For exact analysis five acres of land is considered as reference and consumed energy is observed in terms of $\mathrm{mA}$. In proposed method maximum number of sensors for five acres of land is taken as 900 and these 900 sensors will cover the entire area within the allocated time interval. From Figure 5 it can be observed that corresponding time intervals for 100 , $300,500,700$, and 900 sensors will be $165,250,400,525$, and 600 , respectively.

As mentioned above for all 900 sensors which are divided in five acres of land energy consumption will be monitored and it is compared with existing method [9]. From comparative analysis it can be seen that the proposed method outperforms the existing method in terms of energy. For example if the number of sensors is 500 and predicted time for monitoring load is 400 seconds then energy that is consumed by sensors will be $157 \mathrm{~mA}$ whereas for the same number of sensors and loads the energy consumed by existing method is equal to $200 \mathrm{~mA}$ which is much higher than proposed method. Therefore, the proposed method can be able to reduce the energy that is wasted and can be able to save much energy during sensing operations. 


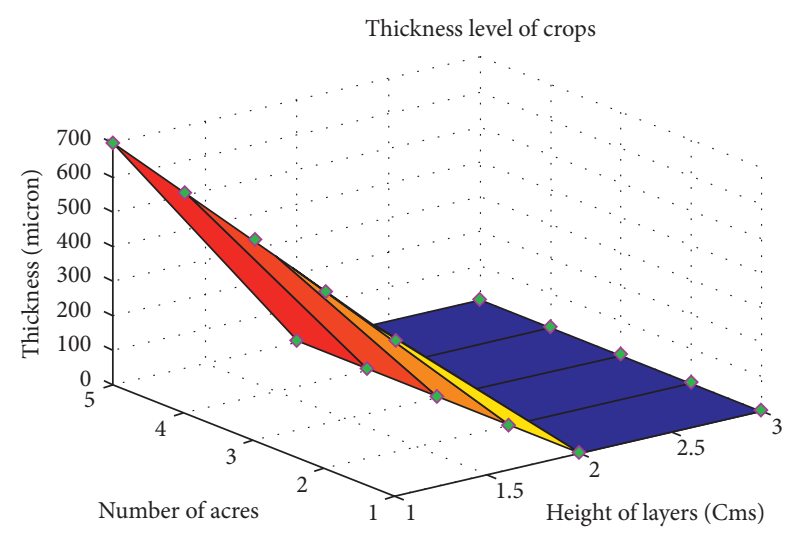

Figure 4: Level of thickness.

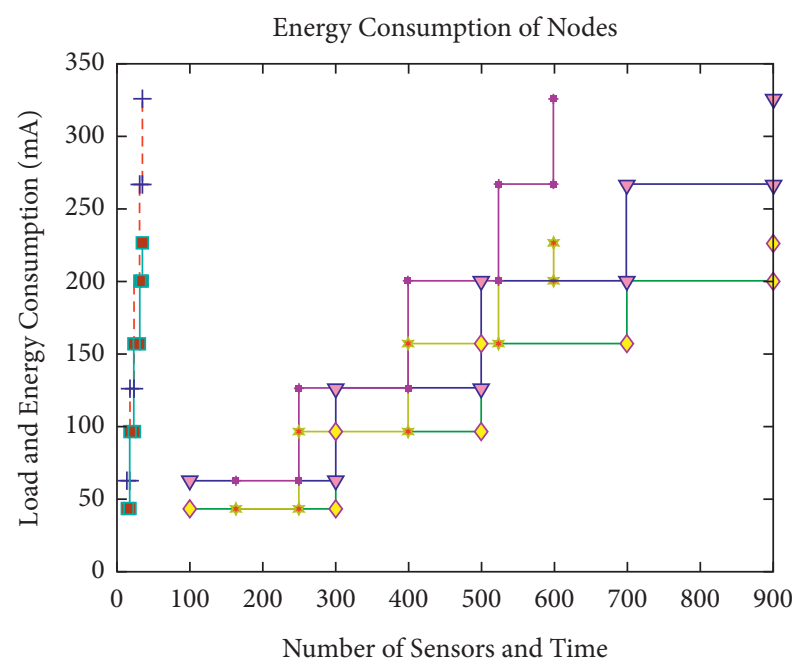

Figure 5: Energy consumption of sensors.

4.5. Scenario 5. After observing the output values of all necessary parameters it is necessary to check cost of implementation of sensors and its corresponding hardware cost because during real time implementation all fragments will be separately distributed to crofters. If cost of implementation is lesser then all individuals can purchase it from resident shops. Therefore by considering the number of sensors and its hardware components total values of cost are simulated and it is compared with existing method [9] as shown in Figure 6.

It can be observed in Figure 6 that total cost of implementation which is measured in dollars is much lesser for the proposed method because appropriate sensors are installed at necessary places in the agricultural field. But for existing method to measure the same amount of parameters larger number of sensors is needed; therefore cost of implementation is much higher. For example if number of sensors is equal to 300 and 500 total cost of implementation for proposed method will be $125 \$$ and $212 \$$, respectively. But for existing method [7] the cost of implementation will be much higher which is equal to $143 \$$ and $235 \$$, respectively. Thus feasible sensors are implemented in the proposed method for attaining much effective cost of implementation. Table 4 is a comparison analysis of energy consumption and total cost.

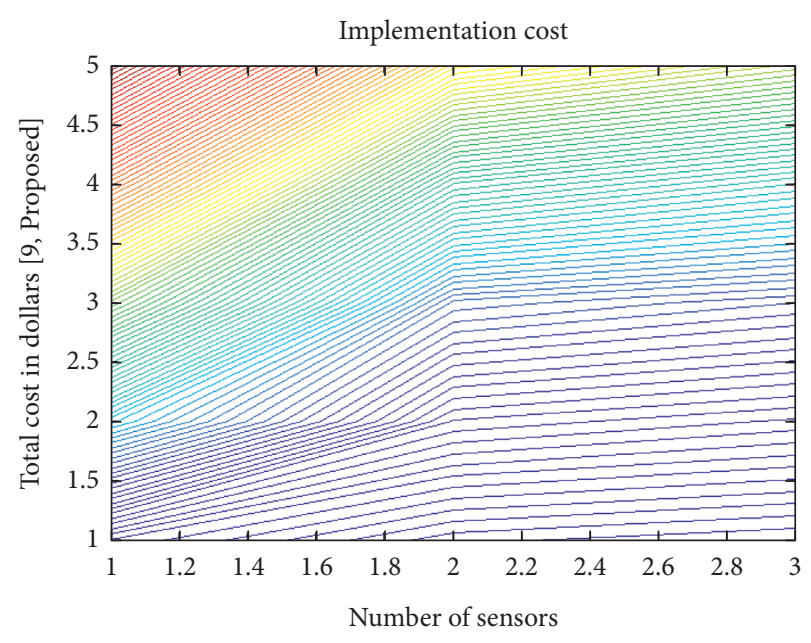

FIgURE 6: Total cost of implementation.

TABLE 4: Comparison analysis.

\begin{tabular}{lcc}
\hline Metrics & Proposed & Existing \\
\hline Energy consumption & $157 \mathrm{~mA}$ & $200 \mathrm{~mA}$ \\
Total cost & $\$ 125-212$ & $\$ 143-235$ \\
\hline
\end{tabular}

\section{Conclusions}

Smarter monitoring and control technologies for budding harvests are necessary; all major parameters that affect the farming level in both rural and urban areas are discussed in this article. If such parameters are monitored appropriately then growth level of all crops in rural areas will be much higher and as a result there will be growth in income level of farmers. Also, if fledgling people are involved in such monitoring process then it will be much easier to grow the crops as illusory; therefore, for this purpose the authors have considered sensor based technology which is implemented with HTTP protocol. In addition, for optimizing the ideals SPA algorithm is applied in proposed formulation. The proposed method provides a profound perception in the process of agriculture and compares all existing methods. It has been found that only few authors have focused on monitoring major parametric values where simulated results prove that proposed method outperforms all existing methods in terms of parametric values. In the future the same technique can be extended to drone based technology with Artificial Intelligence (AI) or Machine Learning (ML) technique where cost of implementation should be reduced.

\section{Data Availability}

The datasets used and/or analyzed during the current study are available from the corresponding author on reasonable request.

\section{Conflicts of Interest}

There are no conflicts of interest. 


\section{Acknowledgments}

The authors gratefully acknowledge the Deanship of Scientific Research, King Khalid University (KKU), Abha-Asir, Kingdom of Saudi Arabia, for funding this research work under the Grant no. RGP.2/58/42.

\section{References}

[1] M. Ayaz, M. Ammad-Uddin, Z. Sharif, A. Mansour, and E.-H. M. Aggoune, "Internet-of-things (IoT)-based smart agriculture: toward making the fields talk," IEEE Access, vol. 7, Article ID 129551, 2019.

[2] A. Ullah, S. Aktar, N. Sutar, R. Kabir, and A. Hossain, "Cost effective smart hydroponic monitoring and controlling system using IoT," Intelligent Control and Automation, vol. 10, no. 4, pp. 142-154, 2019.

[3] A. Kumar, R. A. Devi, P. Sindhusha, and M. R. Marak, "A review on scope and potentiality of vertical farming in India a review on scope and potentiality of vertical farming in India," Journal of Pharmacognosy and Phytochemistry, vol. 9, 2020.

[4] A. R. Chaudhry and V. P. Mishra, "A comparative analysis of vertical agriculture systems in residential apartments, 2019," in Proceedings of the Advances in Science and Engineering Technology International Conferences (ASET) 2019, pp. 1-5, UAE, 2019.

[5] M. I. H. Bin Ismail and N. M. Thamrin, "IoT implementation for indoor vertical farming watering system," in Proceedings of the 2017 International Conference on Electrical, Electronics and System Engineering (ICEESE), pp. 89-94, Kanazawa, Japan, November 2017.

[6] A. G. Nair, A. Chacko, G. Mohan, and T. K. Francis, "Smart vertical farming using hydroponics," Journal of Electrical and Electronic Engineering, vol. 1, pp. 14-17, 2015.

[7] M. Jegadeesh and J. Verapandi, "An innovative approach on vertical farming techniques," International Journal of Agriculture Environmental and Science, vol. 1, pp. 1-5, 2014.

[8] K. Al-Kodmany, "The vertical farm: a review of developments and implications for the vertical city," Buildings, vol. 8, 2018.

[9] F. Kalantari, O. Mohd Tahir, A. Mahmoudi Lahijani, and S. Kalantari, "A review of vertical farming technology: a guide for implementation of building integrated agriculture in cities," Advanced Engineering Forum, vol. 24, pp. 76-91, 2017.

[10] S. Tabassum and A. Hossain, "Design and development of weather monitoring and controlling system for a smart agro (farm)," Intelligent Control and Automation, vol. 9, no. 3, pp. 65-73, 2018.

[11] V. Kishorebabu and R. Sravanthi, "Real time monitoring of environmental parameters using IOT," Wireless Personal Communications, vol. 112, no. 2, pp. 785-808, 2020.

[12] A. Jain and Y. Gupta, "Automated system for monitoring smart farms," International Journal of Modern Trends in Engineering and Research, vol. 7, pp. 32-38, 2020.

[13] A. Nayyar and V. Puri, "Smart farming: IOT based smart sensors agriculture stick for live temperature and moisture monitoring using arduino, cloud computing \& solar technology," in Proceedings of the The International Conference on Communication and Computing Systems (ICCCS-2016), pp. 673-680, Gurgaon, India, November 2016.

[14] S. Lin and L. Zhang, "A mathematical model for vertical column farming system design of leafy vegetable crops," Journal of Physics: Conference Series, vol. 1096, 2018.

[15] M. Sadik Tasrif Anubhove, N. Ashrafi, A. M. Saleque, M. Akter, and S. U. Saif, "Machine learning algorithm based disease detection in tomato with automated image telemetry for vertical farming, 2020," in Proceedings of the 2020 International Conference on Computational Performance Evaluation (COMPE), pp. 250-254, Shillong, India, July 2020.

[16] W. Marchant and S. Tosunoglu, "Robotic implementation to automate a vertical farm system," in Proceedings of the 30th Florida Conference on Recent Advances in Robotics, pp. 11-12, Florida Atlantic University, Boca Raton, FL, USA, 2017, http:// public.eng.fau.edu/design/fcrar2017/papers/ RoboticImplementation.pdf.

[17] B. Franchetti, V. Ntouskos, P. Giuliani, T. Herman, L. Barnes, and F. Pirri, "Vision based modeling of plants phenotyping in vertical farming under artificial lighting," Sensors, vol. 19, pp. 1-21, 2019.

[18] I. A. Lakhiar, G. Jianmin, T. N. Syed, F. A. Chandio, N. A. Buttar, and W. A. Qureshi, "Monitoring and control systems in agriculture using intelligent sensor techniques: a review of the aeroponic system," Journal Sensors.vol. 2018, Article ID 8672769, 18 pages, 2018.

[19] T. Wasson, T. Choudhury, S. Sharma, and P. Kumar, "Integration of RFID and sensor in agriculture using IOT," in Proceedings of the 2017 International Conference on Smart Technologies for Smart Nation (Smart Tech Con), pp. 217-222, Bengaluru, India, Auguest 2017. 